\title{
Coherent Phase Argument for Inflation
}

\author{
Scott Dodelson \\ NASA/Fermilab Astrophysics Center, Fermi National Accelerator Laboratory, Batavia, \\ IL 60510-0500 \\ Department of Astronomy \& Astrophysics, The University of Chicago, Chicago, IL 60637-1433
}

\begin{abstract}
Cosmologists have developed a phenomenally successful picture of structure in the universe based on the idea that the universe expanded exponentially in its earliest moments. There are three pieces of evidence for this exponential expansion - inflation - from observations of anisotropies in the cosmic microwave background. First, the shape of the primordial spectrum is very similar to that predicted by generic inflation models. Second, the angular scale at which the first acoustic peak appears is consistent with the flat universe predicted by inflation. Here I describe the third piece of evidence, perhaps the most convincing of all: the phase coherence needed to account for the clear peak/trough structure observed by the WMAP satellite and its predecessors. I also discuss alternatives to inflation that have been proposed recently and explain how they produce coherent phases.
\end{abstract}

\section{OVERVIEW}

Over the last several years, we have gradually been accumulating evidence for a remarkable theory of the early universe. This theory now accounts for the observed structure in the universe by invoking new fundamental physics at very high energy scales. The theory is so elegant and simple that it contains just a handful of free parameters. It is outlined in Figure 1, which indicates how perturbations generated during inflation evolve with time. The observations today of anisotropies in the radiation and inhomogeneities in the matter therefore bear the imprint of:

- the potential of the field(s) which drive(s) inflation

- the abundances of different types of matter in the universe (baryons, which interact with radiation; dark matter, which does not; and neutrinos, which can freestream out of overdense region): $\Omega_{b}, \Omega_{m}$, and $\Omega_{v}$

- late time phenomena such as dark energy (parametrized by abundance $\Omega_{d e}$ and equation of state $w$ ) and reionization

The most important observations confirming this model come from two segments of the electromagnetic spectrum. First, radio receivers have measured the cosmic microwave background (CMB) to exquisite precision. Second, optical telescopes have captured light from distant galaxies and quasars which tell us about the matter distribution both around those objects [1, 2] and along the line of sight to us [3]. They have also received light from distant objects such as galaxies and supernovae, allowing us to measure distances and fill in a modern Hubble diagram [4, 5]. 


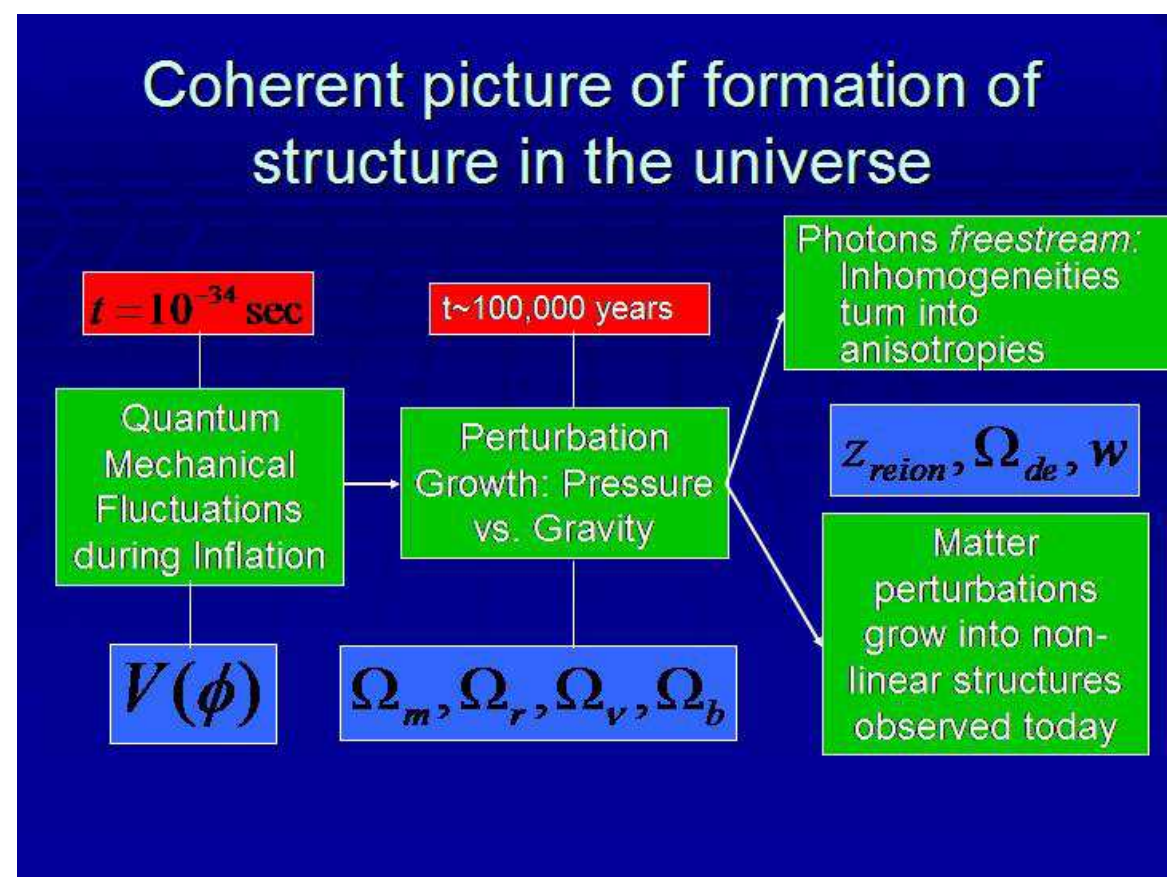

FIGURE 1. Outline of the evolution of structure in the universe. Perturbations are generated at very early times during inflation (determined by the potential $V$ of the field $\phi$ which drives inflation), start to evolve under the combined influence of pressure and gravity when the universe is of order $10^{5}$ years old, and then bifurcate into inhomogeneities in matter (which continue to grow due to gravity) and anisotropies in the radiation (which remain constant).

Here I will focus on one observation and one aspect of the model. Anisotropies in the CMB were first detected in 1992 by the COBE satellite [6]; were probed extensively in the ensuing decade by more than thirty smaller scale experiments [7]; and have now been mapped exquisitely by the WMAP satellite [8]. These observations have been celebrated for measuring cosmological parameters to unprecedented accuracy (e.g. [9, 10]), and I will briefly describe the progress on this front in $\$ 5$. But, for the most part, I want to focus on how the signal seen in the $\mathrm{CMB}$ is smoking gun evidence for the theory of inflation.

\section{INFLATION}

The theory of inflation was introduced over twenty years ago [11] to solve some of the problems of the classical cosmology. For many years, progress was limited to theory and to addressing the question of whether the density in the universe is indeed equal to the critical density as inflation seems to predict. By far the most important, or at least the most testable, aspect of inflation though is its mechanism for producing small perturbations about a smooth background. These perturbations can be measured as long as we account for their (straightforward) evolution after inflation ends. 


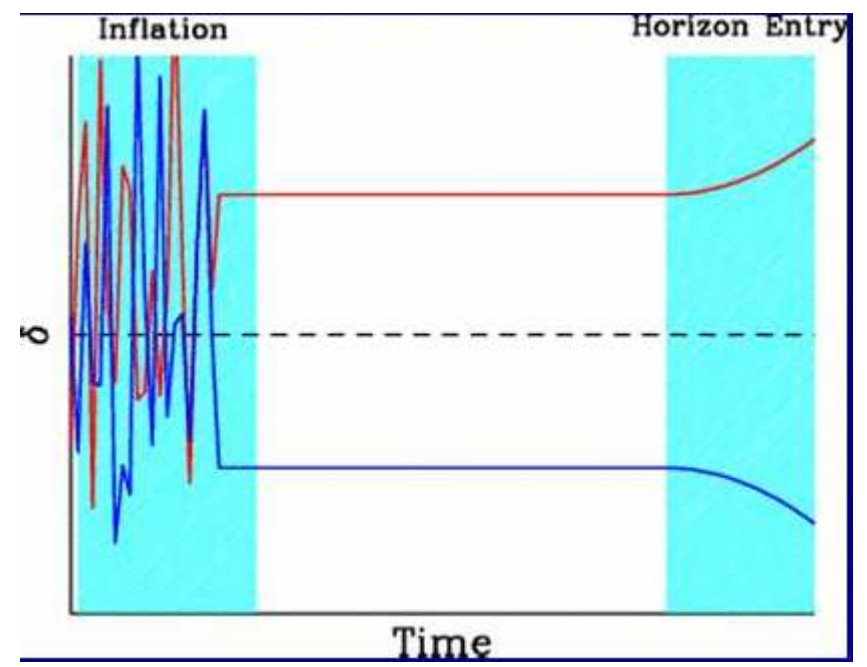

FIGURE 2. Evolution of the amplitudes of two Fourier modes with the same wavelength. After inflation, modes remain constant until they re-enter the horizon. After re-entry, they evolve under the competing influences of pressure and gravity.

It is well known that inflation produces perturbations characterized by a HarrisonZel'dovich spectrum [10, 12]. This means that the amplitude of a particular Fourier mode is drawn from a distribution with mean equal to zero and variance given by

$$
\left\langle\tilde{\delta}(\mathbf{k}) \tilde{\delta}^{*}\left(\mathbf{k}^{\prime}\right)\right\rangle=(2 \pi)^{3} \delta^{3}\left(\mathbf{k}-\mathbf{k}^{\prime}\right) P(k)
$$

where $\delta$ is the fractional overdensity with power spectrum $P(k)$ proportional to $k^{n}$. A Harrison-Zel'dovich spectrum corresponds to $n=1$, and most inflationary models predict something very close to this. You might think then that the shape of the power spectrum can be measured in observations, and this is what convinces us that inflation is right. Well, it is true that we can measure the power spectrum, both of the matter and of the radiation, and it is true that the observations agree with the theory. But this is not what tingles our spines when we look at the data.

Rather, the truly striking aspect of perturbations generated during inflation is that all Fourier modes all have the same phase. To understand what this means and how it develops, consider Fig. 2 which shows a cartoon view of the evolution of the amplitudes of two Fourier modes. Both oscillate quantum mechanically during inflation. Before inflation ends, though, both leave the horizon, that is, their wavelengths get stretched so much that no causal physics can alter them ${ }^{1}$. Once this happens, their amplitudes remain constant. They stay constant up until the time much later on (for modes of interest this might typically happen when the universe is 100,000 years old) when the modes reenter the horizon, at which time causal physics can once again affect their amplitudes. The crucial point here is that as the modes re-enter the horizon, $\dot{\delta}$ is small. If we think of each Fourier mode as a linear combination of a sin and a cos mode, inflation excites

\footnotetext{
${ }^{1}$ Technically this occurs when the wavelength of the mode becomes greater than the Hubble radius, $c / H$.
} 


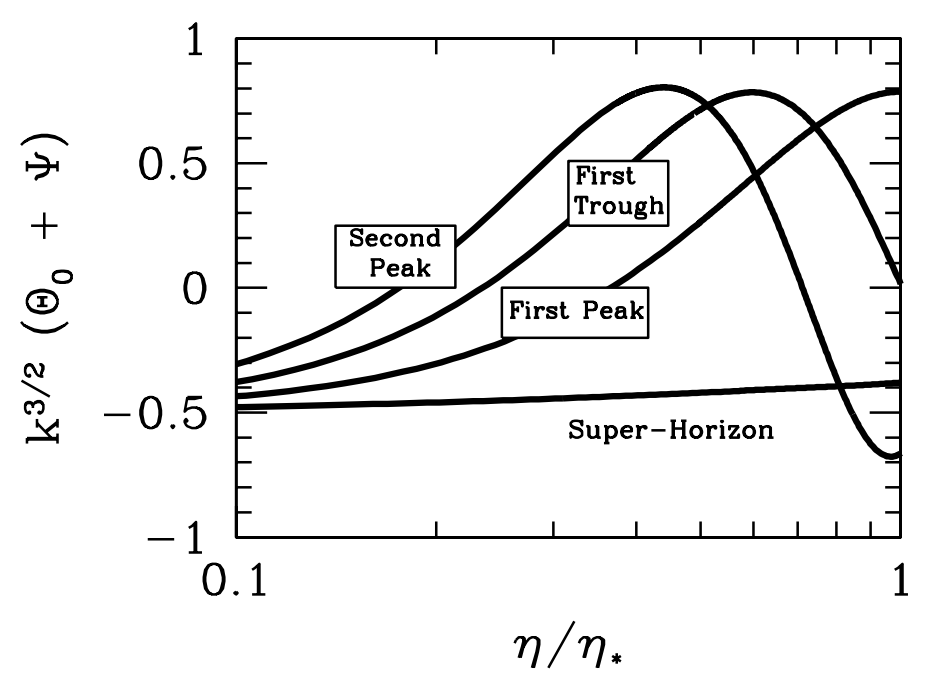

FIGURE 3. Evolution of four Fourier modes of the temperature of the radiation as a function of conformal time $\eta$ ( $=\eta_{*}$ at recombination). Largest scale mode (labeled "Super-Horizon") is still constant at recombination. A slightly smaller scale mode (labeled "First peak") has begun its acoustic oscillation, and has maximal amplitude at recombination. An even smaller scale mode began oscillating earlier; its amplitude is zero at recombination. The smallest scale mode shown here ("Second Peak") has gone through one full oscillation, so its amplitude will be at a maximum. From [10].

only the cos modes. It is difficult to envision any other theory with this striking feature.

\section{ACOUSTIC OSCILLATIONS}

How do perturbations evolve once they re-enter the horizon? A cartoon version of the equation governing them is

$$
\ddot{\delta}-c_{s}^{2} \nabla^{2} \delta=F
$$

where $c_{s}$ is the sound speed and $F$ is a forcing function due to gravity. The perturbations obey the wave equation as one expects physically: a region which is very overdense is driven by gravity to become more overdense, but driven toward the average density by pressure.

At this point, you might come to the conclusion that the spectrum of anisotropies in the radiation today will exhibit a series of peaks and troughs just as a guitar string produces a series of higher harmonics. In fact, the spectrum of the CMB looks remarkably like that of a guitar string. However, underlying the similarity is a pair of differences which are essential to the argument that inflation is the origin of the perturbations.

A guitar string produces a set of harmonics because it is tied down at its ends. So there are only a discrete set of frequencies at which it can oscillate. There is no such restriction for perturbations in the early universe, so why do we see anisotropies at certain frequencies but not at others? 

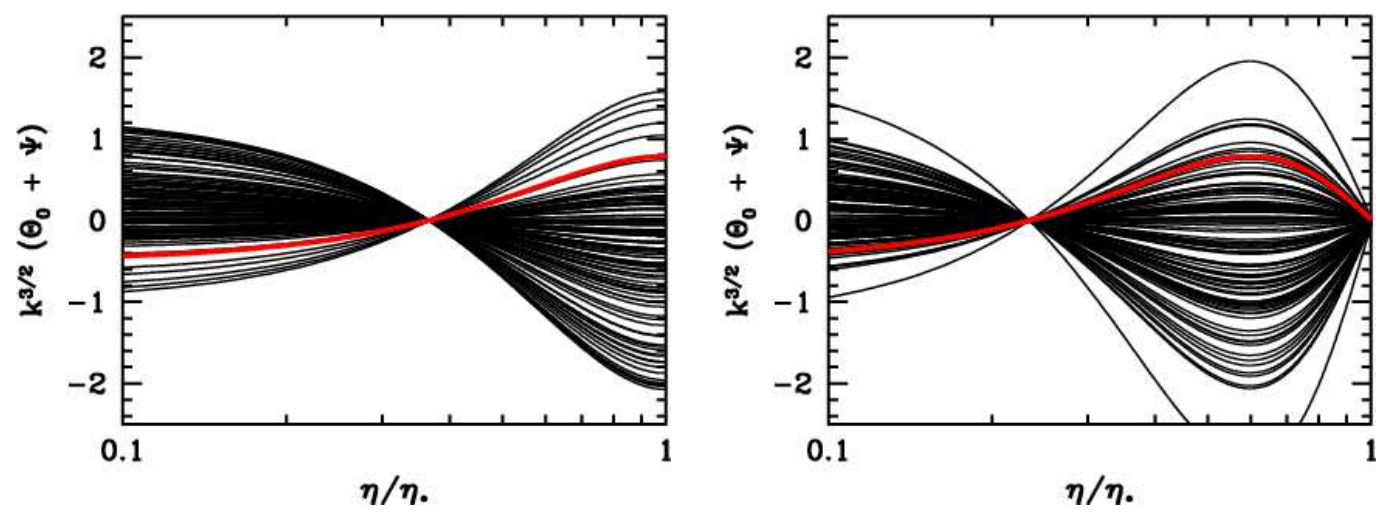

FIGURE 4. The evolution of an infinite number of modes all with the same wavelength. Left panel shows the wavelength corresponding to the first peak, right to the first trough. Although the amplitudes of all these different modes differ from one another, since they start with the same phase, the ones on the left all reach maximum amplitude at recombination; the ones on the right all go to zero at recombination.

To understand this, consider Fig. 3] which shows the evolution of four Fourier modes in the time leading up to recombination ${ }^{2}$. The mode with the largest wavelength cannot be affected by causal physics so its amplitude remains constant. Smaller scale modes have entered the horizon, and so have begun their acoustic oscillations. The smaller the wavelength of a mode, the earlier it will have entered the horizon, and the more oscillations it will have undergone by the time of recombination. Thus, the amplitude of the mode labeled "First Peak" is maximal at recombination, and we expect to see large anisotropies on angular scales which subtend this distance (roughly a degree). The mode labeled "First Trough" has oscillated for a longer time though, and its amplitude is zero at recombination. Therefore, we expect very small anisotropies on the corresponding angular scales. And on it goes, a succession of peaks and troughs present not because no excitations are allowed at the frequencies in the troughs (as is the case for the guitar string). Rather, perturbations are present at all wavelengths, but we happen to see only some of them, depending on the phase of the oscillation at recombination.

It is now very important to remember that there are many, many modes with nearly identical wavenumbers. Think of the number of arrows that can point from the center of a sphere to a fixed radius, keeping in mind that two arrows can be placed infinitesimally close to each other. In fact, since the universe is effectively infinite, there are an infinite number of modes for any wavenumber. All of these get excited during inflation and we must sum over all of them to compute the anisotropy amplitude at a given scale. Thus, when I drew the single line corresponding to the "First Peak" mode in Fig. 3, this was really shorthand for an infinite number of modes all with different amplitudes, as in Fig. 4. The amplitudes may differ, but as Fig. (4)shows, the phases are all the same. All modes enter the horizon with constant amplitude. Thus, all modes with the "First Peak" wavenumber have maximal amplitude (left panel in the figure) at recombination: they

${ }^{2}$ After recombination, photons freestream though the universe, so we see their distribution today as it was at the time of recombination. 

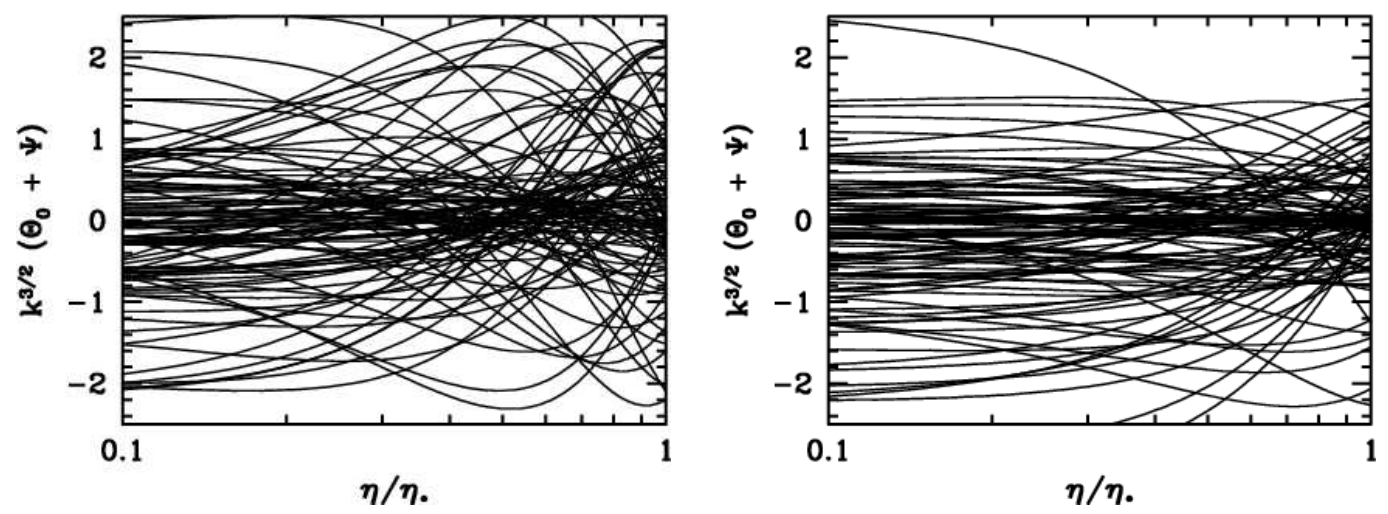

FIGURE 5. Modes corresponding to the same two wavelengths (First Peak and First Trough) as in Fig. 4 but this time with initial phases scrambled. The anisotropies at the angular scales corresponding to these wavelengths would have identical rms's if the phases were random.

have all undergone half an oscillation, so their sign simply changes. Similarly, all modes corresponding to "First Trough" have gone through $3 / 4$ of an oscillation"; since they all are cosine modes and $\cos (3 \pi / 2)=0$, all have zero amplitude at recombination (right panel).

Contrast this with the situation one might otherwise expect, random phases, as depicted in Fig. 5] If the phases were truly random, so that both the sine and cosine modes were excited, then at recombination, there would not be any difference at all between the rms amplitudes of the First Peak and First Trough wavenumbers. So we would not see a sequence of peaks and troughs in the anisotropy spectrum today. We would see simply a flat spectrum with no features. If not for inflation, we would see a flat spectrum. How else could the phases have been set well before the modes of interest entered the horizon?

Therefore, when we look at the anisotropy spectrum recently measured by WMAP [8] and we see the first and second peaks and troughs very clearly (Fig. 6), we are really observing inflation doing the work of coordinating the phases of all Fourier modes. Without this coherence, there would be no peaks and troughs.

\section{POLARIZATION}

The bottom panel of Figure 6 shows the cross-correlation between the temperature and polarization anisotropies. This cross-correlation was first detected by the DASI experiment in late 2002 [13], so our measurements of polarization are much less established than those of temperature. Yet the WMAP results already are a crucial part of the coherence argument for inflation. The peaks and troughs in the anisotropy spectrum all are on angular scales smaller than a degree $(l>200)$; all of these scales were within the

\footnotetext{
${ }^{3}$ You might expect the mode which has gone through $1 / 4$ of a full oscillation to be the first trough. However, there are other effects (the dipole and the Integrated Sachs-Wolfe effect) which fill in this trough.
} 


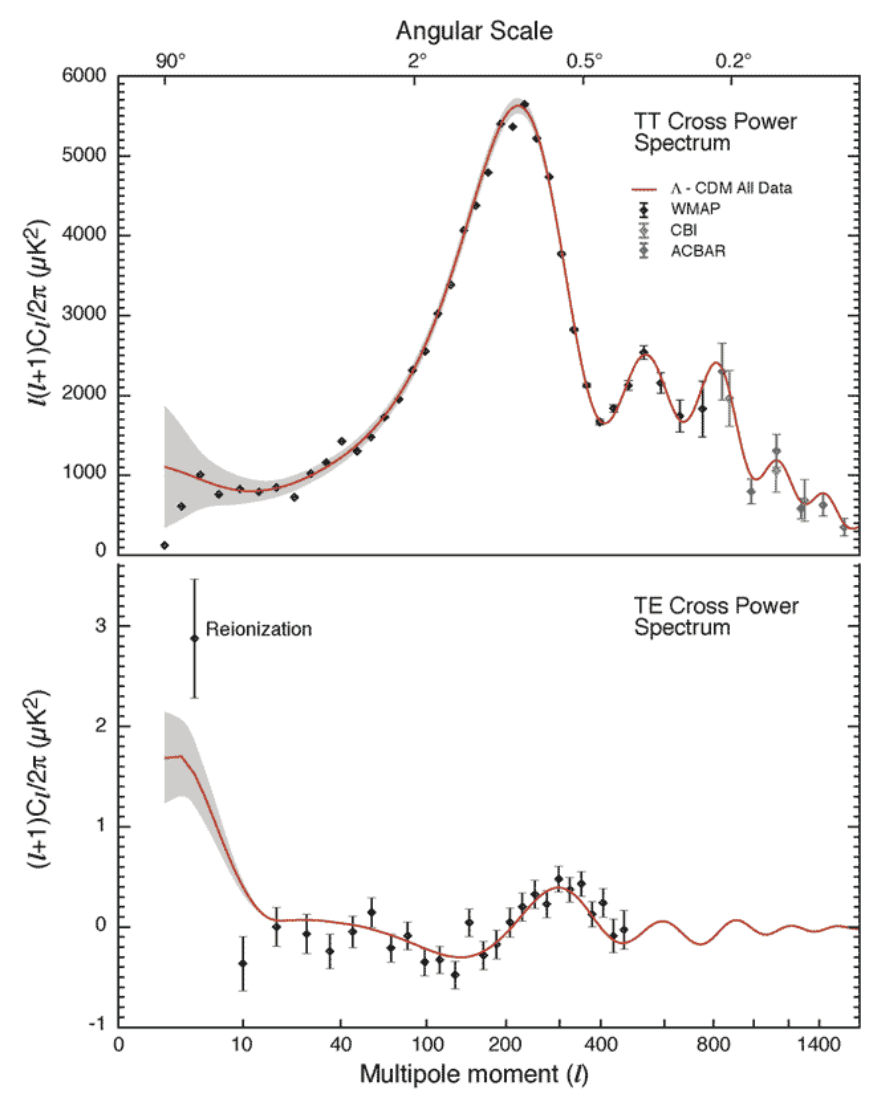

FIGURE 6. Top panel: Temperature anisotropies in the CMB as a function of angular scale [8] (smaller scales toward the right). The series of peaks and troughs is a clear indication of phase coherence, presumably coordinated during inflation. Bottom panel: Cross correlation between the temperature and the polarization as a function of angular scale. The anti-correlation at $100<l<200$ and positive correlation from $200<l<400$ are also due to phase coherence generated during inflation.

horizon at the time of recombination. So you might imagine that one could concoct a theory of structure formation which obeyed causality and still managed to produce only the cosine modes. If you could concoct such a theory [14, 15], then you could explain the peaks and troughs without appealing to inflation. It seems unlikely, but it is at least logically possible.

This logical possibility evaporates when confronted with the polarization data. In particular, the negative cross-correlation between temperature and polarization on scales $100<l<200$ is also the result of phase coherence, as we will shortly see, and the scales involved were not within the horizon at recombination. So there is no causal mechanism that could have produced this anti-correlation: we must appeal to inflation to understand it.

To understand why the temperature and polarization are anti-correlated on scales of order a degree, we first must establish that polarization results from Compton scattering of a radiation field with a quadrupole moment. To see this consider Fig. 7 which depicts incoming radiation in the $z=0$ plane and shows the polarization of the outgoing radiation along the positive $z$-axis. Since the radiation has a quadrupole, incoming 


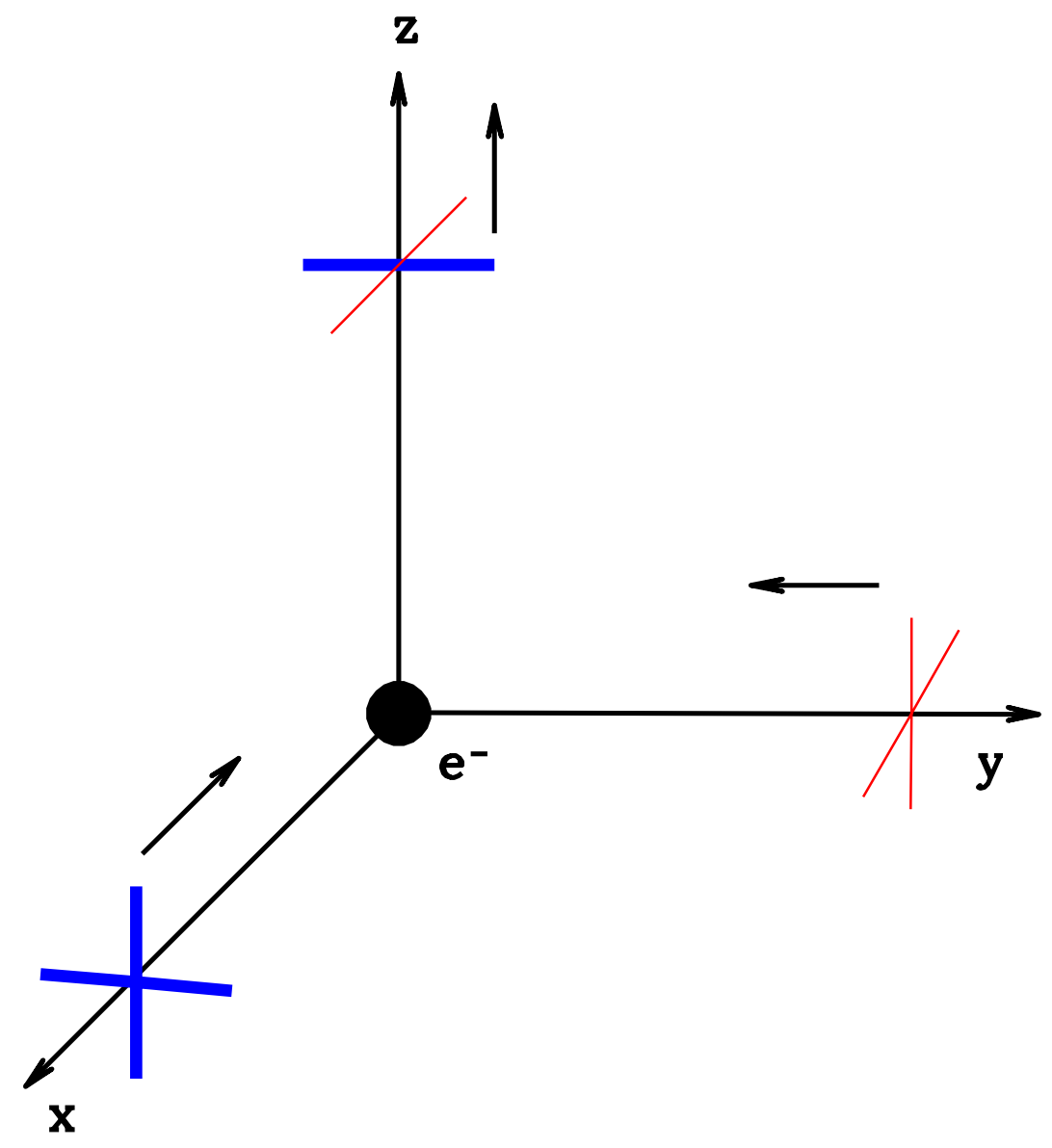

FIGURE 7. Incoming unpolarized radiation along the $x$ - and $y$ - axes produces outgoing polarized radiation along the $z$-axis only if the initial distribution has a non-zero quadrupole moment (figure from [10].)

radiation along the $x$-axis is hotter than that along the $y$-axis. Only the $y$-component of the polarization of the incoming $x$-ray gets transmitted (the $z$-component is parallel to the outgoing direction, and polarization is transverse to the direction of propagation) and only the $x$-component of the incoming $y$-ray gets transmitted. Hence the outgoing $x$-component is cooler than the outgoing $y$-component. A quadrupole in an unpolarized radiation field produces polarized radiation after Compton scattering.

Therefore, the polarization today should be proportional to the quadrupole at the time of recombination. The photons just before recombination are tightly coupled to the electrons. This tight coupling tends to suppress the quadrupole. Consider an observer measuring incoming photons, and for simplicity assume there is only a single plane wave perturbation. When the observer looks perpendicular to the direction along which the density is varying, he sees no perturbation. When he measures along this direction, he measures a Doppler shift, which can be Taylor expanded as

$$
\frac{\delta T}{T}=v+v^{\prime} \lambda_{\mathrm{mfp}}
$$




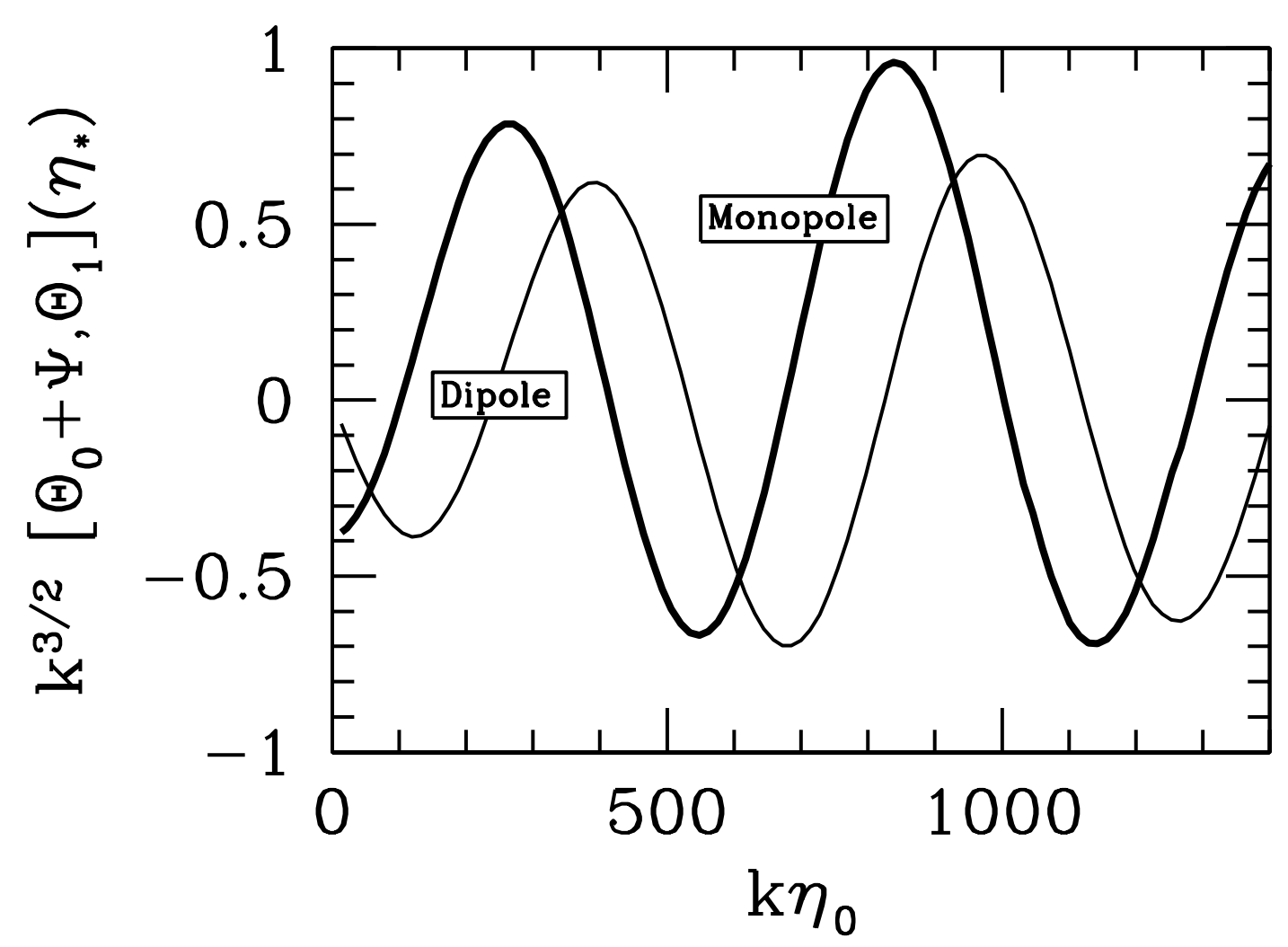

FIGURE 8. The monopole and dipole of the radiation field at recombination as a function of wavenumber $k$ (from [10]). A perturbation with wavenumber $k$ shows up on angular scales $l \sim k \eta_{0}$ where $\eta_{0}$ is (roughly) the distance to the last scattering surface (angle $\theta \sim l^{-1}$ is equal to size of object $k^{-1}$ divided by distance to last scattering surface).

where $v$ is the electron velocity; $v^{\prime}$ its spatial derivative which is of order $v / \lambda$ with $\lambda$ the wavelength of the perturbation; and $\lambda_{\text {mfp }}$ the distance the photon has traveled since it last scattered, the mean free path. The first term here represents the dipole seen by our hypothetical observer, the second the quadrupole. Hence the quadrupole is proportional to $v \lambda_{\mathrm{mfp}} / \lambda$. The quadrupole then is proportional to the electron velocity. The dipole of the radiation is equal to the electron velocity, so the quadrupole is proportional to the dipole right before recombination. The proportionality constant is small, since $\lambda_{\mathrm{mfp}}$ is much smaller than the typical wavelength. Collecting these arguments, we expect

$$
P \simeq D \frac{\lambda_{\mathrm{mfp}}}{\lambda}
$$

where $P$ is the polarization and $D$ the dipole. Incidentally, this explains why measurements of polarization have lagged behind those of temperature anisotropies: the polarization signal is a factor of ten smaller due to the $\lambda_{\mathrm{mfp}} / \lambda$ suppression.

The polarization of the CMB today then is determined by the dipole at recombination. The temperature anisotropies on the other hand are determined by the monopole at 
recombination ${ }^{4}$. The cross-correlation between the temperature anisotropy and the polarization anisotropy then is proportional to the cross-correlation of the monopole and dipole at recombination. How is the monopole related to the dipole at recombination? We can answer this simply by recalling the continuity equation:

$$
\frac{\partial \rho}{\partial t}+\nabla \cdot(\rho \mathbf{v})=0
$$

The velocity then (or equivalently the dipole) is proportional to $\dot{\rho}$, the time derivative of the monopole. This is shown explicitly in Fig. 8 At recombination, this phase difference causes the product of the two to be negative for $100<l<200$ and positive on smaller scales until $l \sim 400$. But this is precisely what WMAP has observed! We have clear evidence that monopole and dipole were out of phase with each other at recombination.

This evidence is exciting for the small scale modes $(l>200)$. Just as the acoustic peaks bear testimony to coherent phases, the cross-correlation of polarization and temperature speaks to the coherence of the dipole as well. It solidifies our picture of the plasma at recombination. The evidence from the larger scale modes $(l<200)$ though is positively stupendous. For, these modes were not within the horizon at recombination. So the only way they could have their phases aligned is if some primordial mechanism did the job, when they were in causal contact. Inflation is just such a mechanism.

\section{COSMOLOGICAL PARAMETERS}

I hope I have convinced you that we now have very good reason to believe in the basic framework of inflation as the seed of structure in the universe. Once we assume this framework, we can go ahead and determine the free parameters in the model. The first and most renowned is that the first peak appears where it should if the universe is flat, so let's assume that the universe is flat. Three of the easiest parameters to measure then are the baryon density $\Omega_{b}$, the matter density $\Omega_{m}$, and the Hubble constant $h$. Actually, as indicated in Fig. 9, the CMB anisotropies are most sensitive to combinations of these parameters.

Here is a rough guide to the sensitivity of the anisotropies to these three cosmological parameters [10].

- Baryon density $\Omega_{b} h^{2}$. The sound speed in Eq. (2) goes down as more baryons are added. The frequency of oscillation thus becomes smaller as the baryon density goes up. A reduced frequency accentuates the effectiveness of the driving force, making the oscillation more asymmetric. The result is that the height of the second peak is much smaller than the height of the first peak when the baryon density is high.

- Matter density $\Omega_{m} h^{2}$. If there is a lot of radiation at recombination, the gravitational potential changes with time, inducing a larger driving force and hence boosting

\footnotetext{
${ }^{4}$ The monopole is what normally thinks of when speaking of the temperature in a given spot. It is the average temperature of all photons hitting that spot coming from all directions.
} 


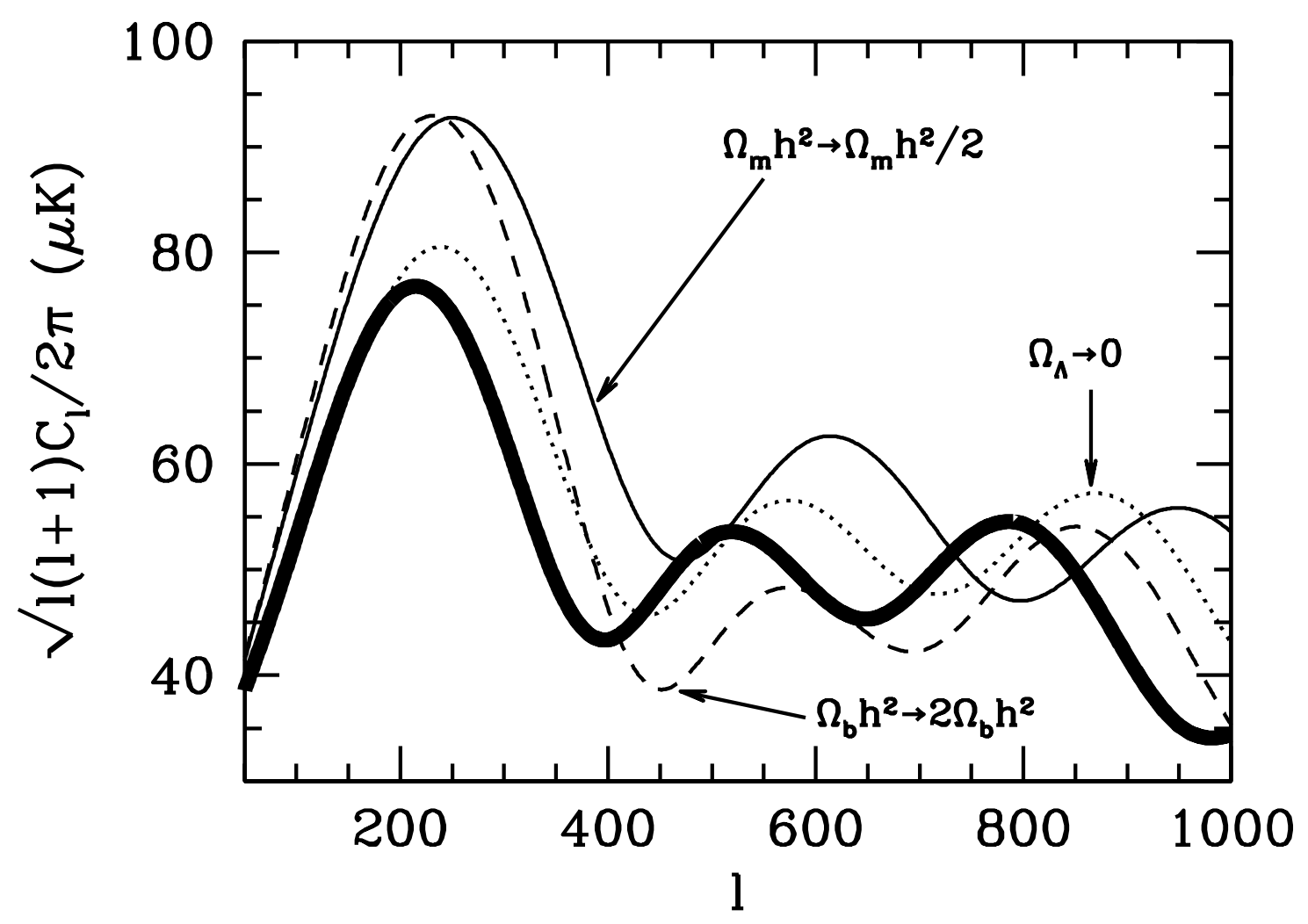

FIGURE 9. Dark solid curve is a model with $70 \%$ cosmological constant and thirty percent baryons. Lighter curves show how the anisotropies change when varying different parameters. Here the total density is set to the critical density. From [10].

anisotropies.

- Cosmological constant $\Omega_{\Lambda}$. The cosmological constant ${ }^{5}$ is a late time effect, so the only impression it leaves on the CMB relates to the way physical scales project onto angular scales; i.e. $\Omega_{\Lambda}$ changes the distance to the last scattering surface, so the curves simply shift horizontally if $\Omega_{\Lambda}$ changes.

The results from WMAP [9] are shown in Fig. 10. We get a sense that the CMB has reduced parameter uncertainties by close to a factor of ten. And this improvement allows us to make several remarkable statements about our universe, based solely on observations of the CMB. First, if one assumes the universe is exactly flat, then the CMB tells us that Hubble constant is $h=0.72 \pm 0.05$, in remarkable agreement with direct determinations [4]. The ratio of the total matter density to the baryonic density is about $6 \pm 1$, which means the $\mathrm{CMB}$ alone requires significant non-baryonic dark matter. Finally, $\Omega_{\Lambda}=1-\Omega_{m}$ is equal to $0.71 \pm 0.07$. The CMB, together with the flatness assumption, requires a cosmological constant, or some form of dark energy. No wonder

\footnotetext{
${ }^{5}$ In a flat universe with constant $\Omega_{m} h^{2}$ and $\Omega_{b} h^{2}$, reducing $\Omega_{\Lambda}$ is equivalent to raising one of the other $\Omega$ 's and reducing the Hubble constant $h$ to keep the products $\Omega_{i} h^{2}$ fixed.
} 


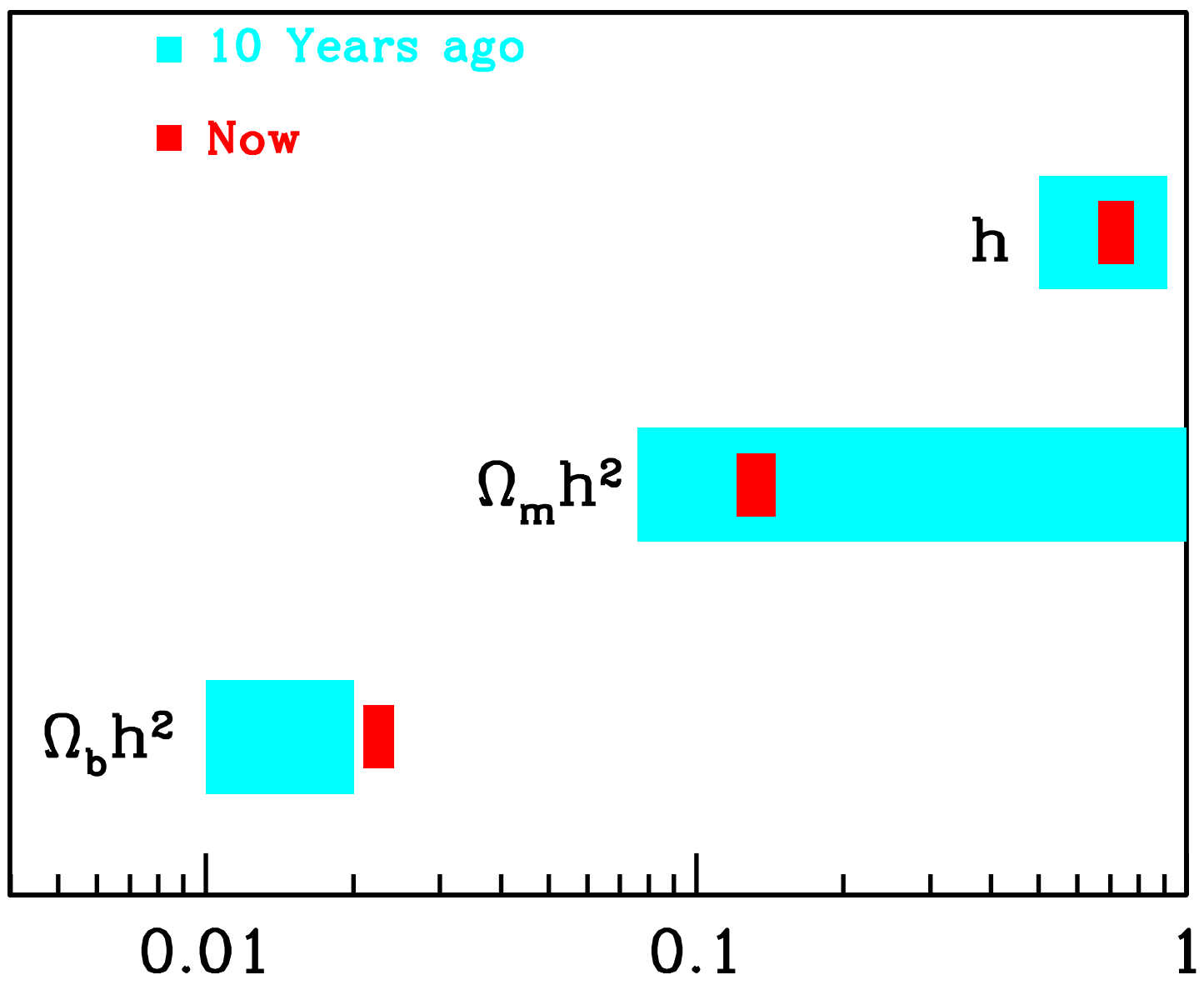

FIGURE 10. Allowed ranges for three cosmological parameters assuming the universe is flat. Light hatched bands are a rough estimate of numbers used ten years ago. The dark solid bands are 1-sigma errors from WMAP [9].

parameter determination has received so much publicity!

\section{ALTERNATIVES}

It is perhaps not surprising that, as the evidence for inflation has firmed up, theoreticians have been working harder than ever to find alternatives to inflation. Here I want to focus on the question of what alternatives are viable in light of the coherent phase argument.

It is instructive to start with two models that don't quite make it. The first is the wellknown class of models with structure seeded by topological defects. The phases of the Fourier modes are not synchronized in defect models, so we do not expect a coherent series of peaks and troughs. This elegant argument was first first advanced by Albrecht et al. [16] as a way of distinguishing defect models from inflation and later confirmed in detailed numerical studies [17, 18].

A second alternative has recently been proposed by Armendariz-Picon and Lim [19]. They note that inflation works by producing perturbations when the modes of interest are 
sub-horizon and then driving these modes to be larger than the horizon. Once outside the horizon, the perturbations freeze-out, i.e. remain constant, until they re-enter the horizon much later around the time of recombination. They point out that really perturbations freeze out once they leave the sound horizon horizon $\left(c_{S} / H\right.$ instead of $\left.H^{-1}\right)$. Thus instead of the Hubble rate remaining roughly constant (as during inflation wherein $c_{s}=1$ ), freeze-out can also be accomplished if the sound speed drops rapidly. This is a clever idea, one that might ultimately be part of a viable alternative. At present though, it doesn't quite work, because - as they point out - inflation is still needed (after perturbation production) to drive the scale beyond the horizon ${ }^{6}$. Another way of saying this is to notice that if not for inflation, the modes of interest would never have been sub-horizon, so nothing could have been effective in producing perturbations.

Indeed, the most basic requirement for coherent phases is that at some point is the distant past (well before recombination), the modes of interest had to be within the horizon. So, the coherent phases requirement is simply a strengthened version of the classical horizon problem. With that in mind, I come to a final class of alternatives which generate perturbations in a variety of ways, but all share the same innovative approach to the horizon problem.

One way to think of the horizon problem is in term of the comoving grid, wherein the wavenumber of any mode remains constant with time. The comoving Hubble radius is $(a H)^{-1}$, which typically increases with time. The classical horizon problem is that, since the comoving Hubble radius monotonically increases in the standard cosmology, these modes must have all been outside the horizon before recombination. The inflationary solution is depicted in Fig. 11 which shows that the necessary requirement is that $a H$ must have increased sometime in the past. Mathematically then we would seem to require

$$
\frac{d}{d t}(a H)=\frac{d^{2} a}{d t^{2}}>0
$$

at some point in the distant past. That is, we seem to require inflation (which can be defined as a period in which $\ddot{a}>0$ ). The alternative models [20] however evade this constraint by using a contracting phase. The requirement that the comoving radius decrease now is $d[-a H] / d t>0$ (since the Hubble radius is $-H^{-1}$ ) or $\ddot{a}<0$. So the horizon problem can be solved and the necessary coherent phases generated if: (i) the universe accelerates while it is expanding (inflation) OR (ii) the universe decelerates while it is contracting.

These alternatives and others are honing in on the question of what we have really learned from the observations. That is, it is no longer sufficient to solve the classical horizon problem. While the scales of interest are sub-horizon, a mechanism is needed to generate perturbations with the proper amplitude and shape and to drive these perturbations beyond the horizon so they freeze out.

\footnotetext{
${ }^{6}$ However, the since scale-invariant perturbations have already been generated, the requirements on inflation are less severe than usual.
} 


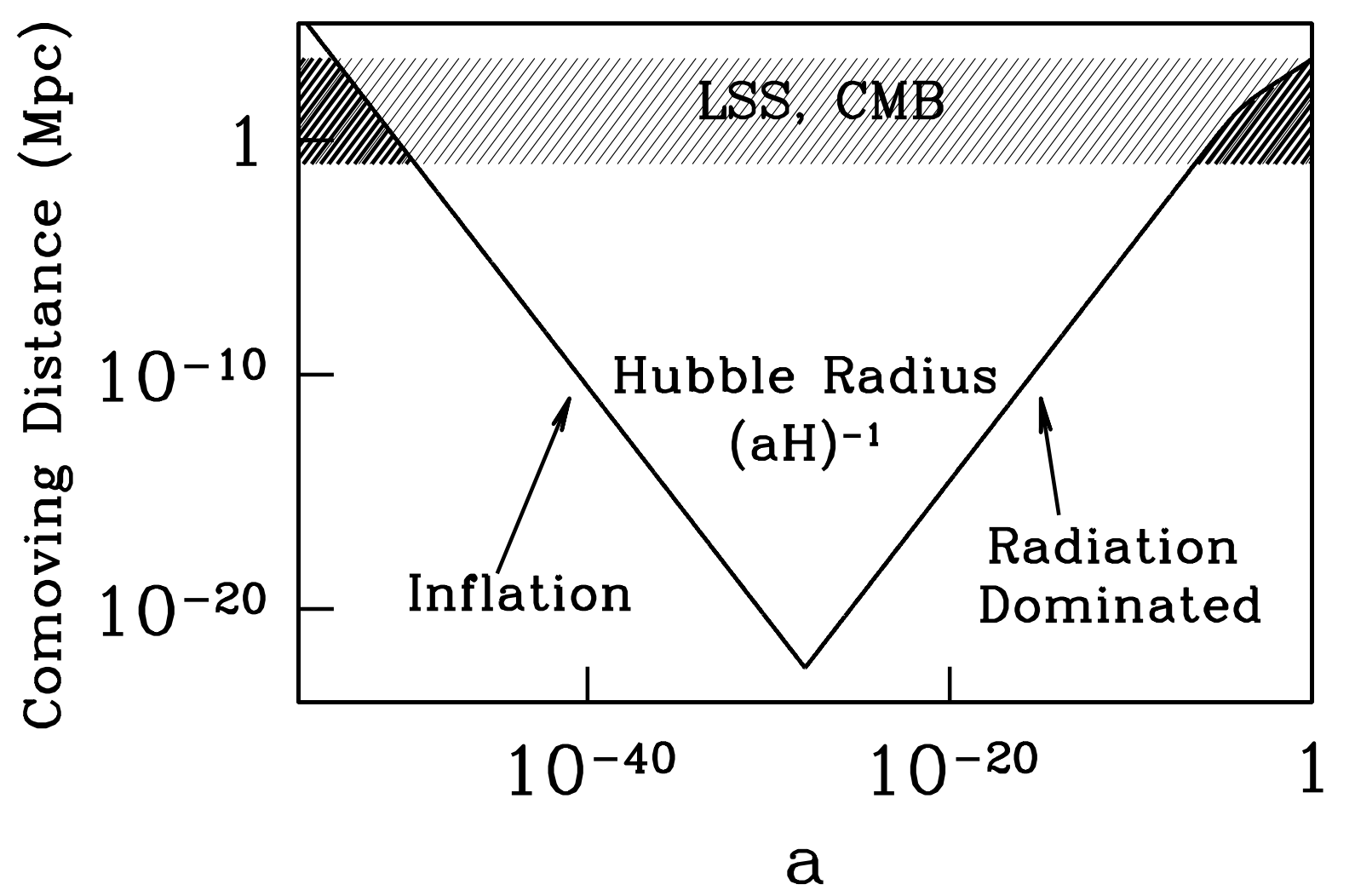

FIGURE 11. Evolution of the comoving Hubble radius in the inflationary picture. During inflation $H$ remains, constant, so the comoving Hubble radius drops as the universe expands exponentially. Thus scales which were initially in causal contact (at $a \sim 10^{-50}$ in the figure) freeze-out. From [10].

\section{CONCLUSIONS}

Detailed observations of the CMB have solidified our confidence in a model of structure formation based on inflation. The most striking evidence for inflation is the phase coherence of the primordial perturbations, which manifests itself in the peaks and troughs of the temperature anisotropies and in the cross-correlation between the temperature and the polarization. Once this framework has been accepted, it is possible to use it and extract cosmological parameters. This parameter estimation suggests that: the universe is flat; there is non-baryonic dark matter; and dark energy dominates the energy budget.

As the observations have improved, theorists have expanded the range of models which can account for them. and proposed new alternatives to inflation. The exciting development is that these alternatives must solve a much more demanding version of the horizon problem. And we are learning more about what precisely is necessary to generate the coherent phases we have so unambiguously observed. 


\section{ACKNOWLEDGMENTS}

This work was supported by the DOE, by NASA Grant NAG5-10842, and by NSF Grant PHY-0079251. I would also like to thank the organizers of the Fourth Tropical Workshop on Particle Physics and Cosmology: R. Volkas, S. Tovey, C. N. Leung, and J. Nieves.

\section{REFERENCES}

1. The 2dF Survey has been released, M. Colless et al., astro-ph/0306581 and the distribution of galaxies in it analyzed in, e.g., D. S. Madgwick et al., astro-ph/0303668.

2. The first data release from the Sloan Digital Sky Survey took place early this year, K. Abazajian et al., astro-ph/0305492 Early analyses of the galaxy distribution include S. Dodelson et al., Astrophys. J. 572, 140 (2001); R. Scranton et al., Astrophys. J. 579, 48 (2002); I. Zehavi et al., astro-ph/0301280.

3. I refer here to gravitational lensing which sheds light on the mass along the line of sight. For a comprehensive review, see M. Bartelmann \& P. Schneider, Phys. Rep. 340, 291 (2001). For the purposes of cosmological parameter determination, perhaps the most important type of lensing has been weak lensing due to large scale structure. The first measurements of this effect were: D. Bacon, A. Refregier, \& R. S. Ellis, Mon. Not. Roy. Ast. Soc. 318, 625 (2000); N. Kaiser, G. Wilson, \& G. Luppino, astro-ph/0003338, L. van Waerbeke et al. Astron \& Astrophys. 358, 30 (2000); D. M. Wittman et al. Nature 405, 143 (2000). For up-to-date summaries, see, e.g., A. Refregier, astro-ph/0307212 and L. van Waerbeke and Y. Mellier, astro-ph/0305089 See e.g. [10] for a quantitative discussion of the ways in which weak lensing probes cosmology.

4. Results from the Hubble Space Telescope are summarized in W. Freedman et al. Astrophys. J. 553, 47 (2001)

5. Results from distant supernovae were reported in A. Riess et al., Astron. J. 116, 1009 (1998); S. Perlmutter et al., Astrophys. J. 517, 565 (1999); J. L. Tonry et al., astro-ph/0305008

6. G. F. Smoot et al., Astrophys. J. 396, L1 (1992)

7. SouthPole91, J. Schuster et al. Astrophys. J. 412, L47 (1991); ARGO, S. Masi et al. Astrophys. J. 463, L47 (1993); WhiteDish, G. B. Tucker et al. Astrophys. J. 419, L45 (1993) FIRS, K. Ganga et al. Astrophys. J. 432, L15 (1994); SouthPole94, J. O. Gunderson et al. Astrophys. J. 443, L57 (1995); CAT96, P. F. Scott et al. Astrophys. J. 461, L1 (1996); MAX, S. Tanaka et al. Astrophys. J. 468, L81 (1996); BAM, G. S. Tucker et al. Astrophys. J. 475, L73 (1997); PYTHON, S. R. Platt et al. Astrophys. J. 475, L1 (1997); SASKATOON, C. B. Netterfield et al. Astrophys. J. 477, 47 (1997); SUZIE, S. E. Church et al. Astrophys. J. 484, 523 (1997); VLA, R. B. Partridge et al. Astrophys. J. 483, 38 (1997); IACB, B. Femenia et al. Astrophys. J. 498, 117 (1998); QMAP, A. de Oliveira-Costa et al. Astrophys. J. 509, L77 (1998); IAC, S. R. Dicker et al. Mon. Not.Roy.Ast.Soc. 309, 750 (1999); MAT, E. Torbet et al. Astrophys. J. 521, L79 (1999); PYTHON5, K. Coble et al. Astrophys. J. 519, L5 (1999); TOCO, A. D. Miller et al. Astrophys. J. 524, L1 (1999); ATCA, R. Subrahmanyan et al. Mon. Not.Roy.Ast.Soc. 315, 808 (2000); BOOMerang97, P. D. Mauskopf et al. Astrophys. J. 536, L59 (2000); CAT99, J. C. Baker et al. Mon. Not.Roy.Ast.Soc. 308, 1173 (2000); MSAM, G. W. Wilson et al. Astrophys. J. 532, 57 (2000); RING, E. M. Leitch et al. Astrophys. J. 532, 37 (2000); TENERIFE, C. M. Gutiérrez et al. Astrophys. J. 529, 47 (2000); VIPER, J. B. Peterson et al. Astrophys. J. 532, L83 (2000); BIMA, K. S. Dawson et al. Astrophys. J. 553, L1 (2001); CBI, S. Padin et al. Astrophys. J. 549, L1 (2001); MAXIMA1, A. T. Lee et al. Astrophys. J. 561, L1 (2001); BOOMerang98, C. B. Netterfield et al. Astrophys. J. 571, 604 (2002); DASI, N. W. Halverson et al. Astrophys. J. 568, 38 (2002); ARCHEOPS, A. Benoit et al., Astron. \& Astrophys. 399, L19 (2003).

8. C. L. Bennett et al., astro-ph/0302207

9. D. N. Spergel et al., astro-ph/0302209

10. S. Dodelson, Modern Cosmology (Academic, San Diego, 2003)

11. A. Guth, Phys. Rev. D23, 347 (1981)

12. S. W. Hawking, Phys. Lett. B 115, 295 (1982); A. A. Starobinsky, Phys. Lett. B117, 175 (1982); A. Guth \& S.-Y. Pi, Phys. Rev. Lett. 49, 1110 (1982); J. M. Bardeen, P. J. Steinhardt, \& M. S. Turner, 
Phys. Rev. D28, 679 (1983)

13. J. Kovac, E. M. Leitch, C. Pryke, J. E. Carlstrom, N. W. Halverson, \& W. L. Holzapfel, Nature 420, $772(2002)$

14. N. Turok, Phys. Rev. D54, 3686 (1996)

15. W. Hu, D. N. Spergel, \& M. White, Phys. Rev. D55, 3288 (1997)

16. A. Albrecht, D. Coulson, P. Ferreira, \& J. Magueijo, Phys. Rev. Lett. 76, 1413 (1996)

17. B. Allen et al., Phys. Rev. Lett. 79, 2624 (1997)

18. U.-L. Pen, U. Seljak, \& N. Turok, Phys. Rev. Lett. 79, 1615 (1997)

19. C. Armendariz-Picon \& E. A. Lim, astro-ph/0307101

20. See, e.g., S. Gratton, J. Khoury, P. J. Steinhardt, \& N. Turok, astro-ph/0301395, J. Khoury, B. A. Ovrut, P. J. Steinhardt, \& N. Turok, Phys. Rev. D66, 046005 (2002); F. Finelli \& R. Brandenberger Phys. Rev. D65, 103522 (2002) 